\title{
EXPONENTIAL GELFOND-KHOVANSKII FORMULA IN DIMENSION ONE
}

\author{
EVGENIA SOPRUNOVA
}

(Communicated by Juha M. Heinonen)

\begin{abstract}
Gelfond and Khovanskii found a formula for the sum of the values of a Laurent polynomial over the zeros of a system of $n$ Laurent polynomials in $(\mathbb{C} \backslash 0)^{n}$. We expect that a similar formula holds in the case of exponential sums with real frequencies. Here we prove such a formula in dimension one.
\end{abstract}

\section{INTRODUCTION}

Recently O. Gelfond and A. Khovanskii discovered a formula for the sum of the values of a Laurent polynomial $g$ over the zeros of a system

$$
f_{1}(z)=\cdots=f_{n}(z)=0, \quad z \in(\mathbb{C} \backslash 0)^{n},
$$

where $f_{i}$ are Laurent polynomials whose Newton polyhedra have generic relative positions $[1,2]$. After an exponential change of variables we obtain a similar formula where $g$ and the $f_{i}$ are now exponential sums with rational frequencies. There is a lot of evidence that this formula continues to hold if the frequencies are real. For example, when $g=1$, the formula follows from combining two results: Gelfond's generalization of Bernstein's theorem [4] and the new formula for mixed volume [5]. In [9] the formula is proved in the case when the frequencies of the exponential sum $g$ are not commensurate with the frequencies of the system.

Here we prove the generalization of the Gelfond-Khovanskii formula to exponential sums with real frequencies in dimension one. Our argument is elementary but it relies on Khovanskii's fewnomial theory [6].

A similar result is obtained by J. Ritt in [8], where he computes the average sum of the real parts of zeros of an exponential sum. Ritt's result can be considered as an exponential generalization of the Vieta formula for the product of zeros of a polynomial.

\section{Algebraic CASE}

Recall that a Laurent polynomial $f$ in one complex variable $z$ is a finite linear combination of monomials with integer exponents:

$$
f(z)=\sum_{k} c_{k} z^{k}, \quad k \in \mathbb{Z}, \quad c_{k} \in \mathbb{C} .
$$

Received by the editors October 25, 2006.

2000 Mathematics Subject Classification. Primary 30C15.

Key words and phrases. Exponential sums, mean value, mean number of zeros.

(C) 2007 American Mathematical Society 
We explain how to compute explicitly the sum of the values of a Laurent polynomial $g$ over the zeros of a Laurent polynomial $f$ in $\mathbb{C} \backslash\{0\}$, counting multiplicities. Our argument in the exponential case is a generalization of the argument that we present here.

Consider a differential form $\omega=g d f / f$. This form has simple poles at the zeros $z_{i}$ of $f$ with the residue equal to $\mu_{i} g\left(z_{i}\right)$ where $\mu_{i}$ is the multiplicity of the root $z_{i}$. Since the sum of the residues of $\omega$ is zero, the sum of the values of $g$ over the zeros of $f$ in $\mathbb{C} \backslash\{0\}$ is equal to $-\operatorname{res}_{\infty} \omega-\operatorname{res}_{0} \omega$.

To compute these residues, we expand $g f^{\prime} / f$ into Laurent series as follows. Let

$$
f(z)=c_{1} z^{k_{1}}+\cdots+c_{n} z^{k_{n}}, \quad k_{1}<\cdots<k_{n}, \quad k_{i} \in \mathbb{Z} .
$$

Put $\tilde{f}=f / c_{1} z^{k_{1}}$. Since $\tilde{f}-1$ contains only positive powers of $z$, we have $|\tilde{f}-1|<1$ in a neighborhood of zero. Therefore, we can expand $1 / \tilde{f}$ into series converging uniformly in this neighborhood:

$$
1 / \tilde{f}=1+(1-\tilde{f})+(1-\tilde{f})^{2}+\cdots .
$$

Since each power of $z$ appears in this series finitely many times, it is a well-defined series in $z$. Multiplying this series by $g f^{\prime} / c_{1} z^{k_{1}}$, we obtain a series for $g f^{\prime} / f$ :

$$
\frac{g f^{\prime}}{f}=\frac{g f^{\prime}}{c_{1} z^{k_{1}}}\left(1+(1-\widetilde{f})+(1-\widetilde{f})^{2}+\cdots\right) .
$$

Therefore, the residue at zero is equal to the coefficient $A_{1}$ of $1 / z$ in this series.

Similarly, denote by $A_{n}$ the coefficient of $1 / z$ in the series

$$
\frac{g f^{\prime}}{c_{n} z^{k_{n}}}\left(1+(1-\widetilde{f})+(1-\widetilde{f})^{2}+\cdots\right)
$$

where this time $\tilde{f}=f / c_{n} z^{k_{n}}$. Then the residue at infinity is equal to $-A_{n}$.

Therefore, the sum of the values of $g$ over the zeros of $f$ in $\mathbb{C} \backslash\{0\}$ is equal to $A_{n}-A_{1}$.

\section{Statement of Result}

We are going to deal with exponential sums with real frequencies, i.e. functions of the form

$$
f(z)=c_{1} \exp 2 \pi \alpha_{1} z+\cdots+c_{n} \exp 2 \pi \alpha_{n} z,
$$

where the coefficients $c_{i}$ are nonzero complex numbers and the frequencies $\alpha_{i}$ are increasing real numbers: $\alpha_{1}<\cdots<\alpha_{n}$. If we factor out $c_{1} \exp 2 \pi \alpha_{1} z$ from $f$, the rest will be close to infinity as $\operatorname{Re} z \rightarrow \infty$ and close to 1 as $\operatorname{Re} z \rightarrow-\infty$. Therefore, all the zeros of $f$ lie in the vertical strip $|\operatorname{Re} z|<B$ for some $B$, where the choice of $B$ depends only on the frequencies $\alpha_{i}$ and the absolute values of the coefficients $c_{i}$.

Let $g$ be an exponential sum with real frequencies. We want to add up the values of $g$ over the zeros of $f$. Since the number of zeros is infinite, the sum over the zeros must be replaced with the result of averaging $g$ over the zeros of $f$ along the imaginary axis. More precisely, let $S(R)$ be the sum of the values of $g$ over those zeros of $f$ (counting multiplicities) whose absolute value of the imaginary part is less than $R$. Since all the zeros of $f$ belong to the vertical strip $|\operatorname{Re} z|<B$, this sum is finite. 
Definition 3.1. The mean value of $g$ over the zeros of $f$ is the limit

$$
M=\lim _{R \rightarrow \infty} \frac{S(R)}{2 R} .
$$

If $g=1, M$ is the mean number of zeros of $f$.

Let $\tilde{f}=f / c_{k} \exp 2 \pi \alpha_{k} z$, where $k$ is either 1 or $n$. The constant term of the exponential sum $\tilde{f}$ is equal to one. Thus we can define the exponential series for $1 / \widetilde{f}$ by the formula

$$
1 / \tilde{f}=1+(1-\tilde{f})+(1-\tilde{f})^{2}+\cdots .
$$

Since each exponent $\exp 2 \pi \alpha z$ appears with a nonzero coefficient in a finite number of terms, the coefficients of these series are well-defined. Let $A_{k}$ be the constant term in the formal product of this series and $g f^{\prime} / c_{k} \exp \left(2 \pi \alpha_{k} z\right)$ for $k=1, n$.

The following theorem is the generalization of the Gelfond-Khovanskii formula to the case of exponential sums in dimension one.

Theorem 3.2. Let $f$ be an exponential sum with real frequencies:

$$
f(z)=c_{1} \exp 2 \pi \alpha_{1} z+\cdots+c_{n} \exp 2 \pi \alpha_{n} z, \quad \alpha_{1}<\cdots<\alpha_{n} .
$$

Let $g$ be another exponential sum with real frequencies. Then the mean value $M$ of $g$ over the zeros of $f$ is equal to

$$
\frac{A_{n}-A_{1}}{2 \pi}
$$

where $A_{n}$ and $A_{1}$ are defined above. In particular, the mean number of zeros of $f$ is equal to $\alpha_{n}-\alpha_{1}$.

Furthermore, the mean value of $g=\exp 2 \pi \alpha z$ over the zeros of $f$ can only be nonzero if $\alpha$ belongs either to the nonpositive semigroup $\mathcal{A}$ generated by the set $\left\{\alpha_{1}-\alpha_{i} \mid i=1 \ldots n\right\}$ or to the nonnegative semigroup $\mathcal{B}$ generated by the set $\left\{\alpha_{n}-\alpha_{i} \mid i=1 \ldots n\right\}$.

\section{The Plan of the PROOF}

We can assume that $f$ does not have zeros on $|\operatorname{Im} z|=R$. We have

$$
M=\lim _{R \rightarrow \infty} \frac{S(R)}{2 R}=\lim _{R \rightarrow \infty} \frac{1}{4 \pi i R} \int_{\Gamma_{R}} \frac{g f^{\prime}}{f} d z,
$$

where the integration is performed in the positive sense over the rectangle $\Gamma_{R}$ bounded by the lines $\operatorname{Re} z=B, \operatorname{Im} z=R, \operatorname{Re} z=-B$, and $\operatorname{Im} z=-R$. Denote the corresponding sides of $\Gamma_{R}$ by $\gamma_{+}, \gamma_{R}, \gamma_{-}$, and $\gamma_{-R}$ (see Figure 4.1).

The proof of Theorem 3.2 will be divided in two parts between the following two sections as follows:

(1) We show that the integral over the horizontal part of $\Gamma_{R}$ is bounded as $R \rightarrow \infty$, and consequently only the integrals over the vertical segments contribute to the mean value.

(2) We compute the contribution to the mean value of the integrals over the vertical segments. 


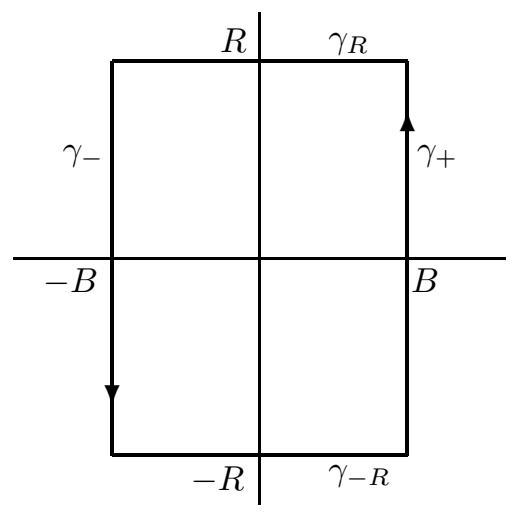

Figure 4.1. Contour $\Gamma_{R}$

\section{The integral over the horizontal part of $\Gamma_{R}$ IS BOUnded}

For each zero of the exponential sum $f(z)=c_{1} \exp 2 \pi \alpha_{1} z+\cdots+c_{n} \exp 2 \pi \alpha_{n} z$ we delete from $|\operatorname{Re} z| \leq B$ an open disk of radius $r>0$ centered at that zero, and we call the resulting region $D_{f}$. In the lemma below we show that $f$ is separated from 0 on $D_{f}$.

Lemma 5.1. For $D_{f}$ defined above there exists $\delta>0$ such that for all $z \in D_{f}$

$$
|f(z)| \geq \delta>0 \text {. }
$$

Proof. Let $\mathcal{F}$ be the family of exponential sums that have the same frequencies $\alpha_{i}$ as $f$ and each of its coefficients $d_{i}$ has the same absolute value as the corresponding $c_{i}$ in $f$. That is, each $h \in \mathcal{F}$ is of the form

$$
h(z)=d_{1} \exp 2 \pi \alpha_{1} z+\cdots+d_{n} \exp 2 \pi \alpha_{n} z,
$$

where $\left|d_{i}\right|=\left|c_{i}\right|$. This family is invariant under translations along the imaginary axis. Due to our choice of $B$, all the zeros of each of the functions in $\mathcal{F}$ belong to $|\operatorname{Re} z|<B$.

For each element $h$ of $\mathcal{F}$ we define $D_{h}$ to be the strip $|\operatorname{Re} z| \leq B$ with deleted open disks of radius $r$ centered at the zeros of $h$. Let

$$
\delta_{h}=\min _{z \in D_{h}}|h(z)|, \quad \delta=\inf _{h \in \mathcal{F}} \delta_{h} .
$$

We need to show that $\delta>0$.

Assume $\delta=0$. It means that there exist a sequence of functions $h_{l} \in \mathcal{F}$ and a sequence of points $z_{l} \in D_{h_{l}}$ such that $h_{l}\left(z_{l}\right) \rightarrow 0$, as $l \rightarrow \infty$. Since $\mathcal{F}$ is invariant under translations along the imaginary axis, we can assume that all $z_{l}$ belong to the compact set

$$
V=\{|\operatorname{Re} z| \leq B,|\operatorname{Im} z| \leq 1\} .
$$

Each $h \in \mathcal{F}$ can be identified with the collection of its coefficients $\left(d_{1}, \ldots, d_{n}\right) \in \mathbb{C}^{n}$. Then the convergence in $\mathbb{C}^{n}$ corresponds to the uniform convergence on $V$. The family $\mathcal{F}$ is a product of $n$ circles and, therefore, is a compact set.

Consider the sequence $\left(h_{l}, z_{l}\right)$ in $\mathcal{F} \times V$. Since $\mathcal{F} \times V$ is compact, we can assume (choosing a subsequence if necessary) that the sequence $\left(h_{l}, z_{l}\right)$ converges to some $\left(h, z_{0}\right) \in \mathcal{F} \times V$. This means that $z_{l}$ converges to $z_{0}$ and $h_{l}$ converges to $h$ uniformly on $V$. Since $h_{l}\left(z_{l}\right) \rightarrow 0$, we have $h\left(z_{0}\right)=0$. 
By the Hurwitz theorem, each function $h_{l}$ with $l$ big enough has a zero in the $\operatorname{disk}\left\{\left|z-z_{0}\right|<\rho\right\} \subset\{|\operatorname{Re} z| \leq B\}$ with $\rho<r / 2$. Therefore, we can find $l$ such that $z_{l}$ belongs to the disk and $h_{l}$ has a zero in this disk. But then the distance between $z_{l}$ and this zero of $h_{l}$ is less than $r$, which contradicts $z_{l} \in D_{h_{l}}$.

This contradiction proves that $\delta>0$.

We will need the following result from the theory of fewnomials, which is a consequence of the complex Rolle theorem.

Theorem $5.2([7])$. Let $f$ be an exponential sum of the form

$$
f(z)=c_{1} \exp 2 \pi \alpha_{1} z+\cdots+c_{n} \exp 2 \pi \alpha_{n} z,
$$

where the coefficients $c_{i}$ are complex numbers and the frequencies $\alpha_{i}$ are increasing real numbers: $\alpha_{1}<\cdots<\alpha_{n}$. Then $f$ has fewer than $n$ zeros in a horizontal strip of width less than $1 /\left(\alpha_{n}-\alpha_{1}\right)$.

Recall that $\gamma_{R} \cup \gamma_{-R}$ is the horizontal part of the contour $\Gamma_{R}$.

Lemma 5.3. The absolute value of the integral

$$
\int_{\gamma_{R}} \frac{g f^{\prime}}{f} d z
$$

is bounded for all $R$ such that there are no zeros of $f$ on $\gamma_{R}$.

Proof. Choose $r<1 / 4 n\left(\alpha_{n}-\alpha_{1}\right)$. For each zero of $f$ delete an open disk of radius $r$ centered at that zero. The projection of these deleted disks to the imaginary axis does not entirely cover the vertical segment

$$
\left\{\operatorname{Re} z=0,|\operatorname{Im} z-R|<\frac{1}{4\left(\alpha_{n}-\alpha_{1}\right)}\right\} .
$$

Indeed, the only disks whose projections could touch this segment are the ones whose center $z$ satisfies

$$
|\operatorname{Im} z-R|<\frac{1}{2\left(\alpha_{n}-\alpha_{1}\right)} .
$$

By Theorem 5.2, there are fewer than $n$ such disks. Hence the length of the projection is less than $2 r n<1 / 2\left(\alpha_{n}-\alpha_{1}\right)$, which is equal to the length of the vertical segment. Therefore, we can choose $R^{\prime}$ on this segment so that $\gamma_{R^{\prime}}$ does not meet the deleted disks. Since by Lemma $5.1 f$ is separated from zero on $\gamma_{R^{\prime}}$, the integral over $R^{\prime}$ is bounded.

It remains to show that the difference of the integrals over $\gamma_{R}$ and $\gamma_{R^{\prime}}$ is bounded. Consider the contour $\gamma$ formed by $\gamma_{R}, \gamma_{R^{\prime}}$, and the vertical segments connecting their endpoints. The integral over $\gamma$ is equal to the sum of the values of $g$ over the zeros of $f$ that lie inside $\gamma$, times $2 \pi i$. This sum is bounded since $f$ has fewer than $n$ zeros inside, and $g$ is bounded on $|\operatorname{Re} z|<B$. Choosing $B$ so big that all the zeros of $f$ belong to $|\operatorname{Re} z|<B / 2$ and requiring that $r<B / 2$, we ensure that the deleted disks do not intersect the vertical part of $\gamma$ and the integral over the vertical segments is bounded. We proved that the integral

$$
\int_{\gamma_{R}} \frac{g f^{\prime}}{f} d z
$$

is bounded. 
This lemma implies that

$$
\lim _{R \rightarrow \infty} \frac{1}{4 \pi i R} \int_{\gamma_{R}} \frac{g f^{\prime}}{f} d z=0 .
$$

Therefore, only the integrals over the vertical part of $\Gamma_{R}$ can contribute to the mean value.

\section{The integral OVer the Vertical Part of $\Gamma_{R}$}

Here we explain how to compute the limit

$$
\lim _{R \rightarrow \infty} \frac{1}{4 \pi i R} \int \frac{g f^{\prime}}{f} d z
$$

where the integration is performed over the vertical part of $\Gamma_{R}$.

We deal with $\gamma_{-}$first. Recall that $f(z)=c_{1} \exp 2 \pi \alpha_{1} z+\cdots+c_{n} \exp 2 \pi \alpha_{n} z$, where $\alpha_{1}<\cdots<\alpha_{n}$. Let $\tilde{f}=f / c_{1} \exp 2 \pi \alpha_{1} z$. Then the constant term of $\widetilde{f}$ is equal to 1 . On $\operatorname{Re} z=-B$,

$$
|\widetilde{f}-1|=\left|\sum_{j=2}^{n} \frac{c_{j}}{c_{1}} \exp 2 \pi\left(\alpha_{j}-\alpha_{1}\right) z\right| \leq \sum_{j=2}^{n} \frac{\left|c_{j}\right|}{\left|c_{1}\right|} \exp \left(-2 B \pi\left(\alpha_{j}-\alpha_{1}\right)\right),
$$

which is less than 1 if $B$ is big enough since $\alpha_{j}>\alpha_{1}$. Therefore, we can expand $g f^{\prime} / f$ into geometric series converging uniformly on $\operatorname{Re} z=-B$ :

$$
g \frac{f^{\prime}}{f}=g \frac{f^{\prime}}{c_{1} \exp 2 \pi \alpha_{1} z}\left(1+(1-\widetilde{f})+(1-\widetilde{f})^{2}+\ldots\right),
$$

which is a series in exponents, since every exponent appears only finitely many times. We integrate this series over $\gamma_{-}$, divide by $4 \pi i R$ and find the limit as $R \rightarrow \infty$ term-by-term.

Notice that only the constant term in this series can give a nonzero limit. If this constant term is $A_{1}$, then the corresponding limit is $-A_{1} / 2 \pi$. If $g(z)=\exp 2 \pi \alpha z$, then the only values of $\alpha$ for which there is a nonzero constant term in the series are those that belong to the nonpositive semigroup $\mathcal{A}$ generated by $\left\{\alpha_{1}-\alpha_{i} \mid i=\right.$ $1, \ldots, n\}$.

It remains to repeat this procedure for $\gamma_{+}$. Let $\widetilde{f}=f / c_{n} \exp 2 \pi \alpha_{n} z$, and expand $g f^{\prime} / f$ into exponential series converging uniformly on $\operatorname{Re} z=B$ :

$$
\frac{g f^{\prime}}{f}=\frac{g f^{\prime}}{c_{n} \exp 2 \pi \alpha_{n} z}\left(1+(1-\widetilde{f})+(1-\widetilde{f})^{2}+\ldots\right) .
$$

If the constant term of this series is $A_{n}$, then the corresponding limit is $A_{n} / 2 \pi$. If $g(z)=\exp 2 \pi \alpha z$, then the only values of $\alpha$ for which there is a nonzero constant term in the series are those that belong to the nonnegative semigroup $\mathcal{B}$ generated by $\left\{\alpha_{n}-\alpha_{i} \mid i=1, \ldots, n\right\}$.

We proved that the mean value $M$ of $g$ over the zeros of $f$ is equal to

$$
\frac{A_{n}-A_{1}}{2 \pi} \text {. }
$$

If $g=1$, it is easy to see that $A_{n}=2 \pi \alpha_{n}$ and $A_{1}=2 \pi \alpha_{1}$. Hence the mean number of zeros of $f$ is equal to $\alpha_{n}-\alpha_{1}$. 


\section{ACKNOWLEDGMENT}

I would like to thank Askold Khovanskii for stating the problem and for his constant attention to this work.

\section{REFERENCES}

[1] O. Gelfond, A. Khovanskii, Newton polyhedra and Grothendieck residues (in Russian), Dokl. Akad. Nauk, 350 (1996), no. 3, 298-300. MR1444043 (98b:32004)

[2] O. Gelfond, A. Khovanskii, Toric geometry and Grothendieck residues, Moscow Mathematical Journal, Vol. 2 (2002), no. 1, 99-112. MR1900586 (2003h:14074)

[3] O. Gelfond, Zeros of systems of quasiperiodic polynomials, FIAN preprint, no. 200 (1978).

[4] O. Gelfond, The mean number of roots of systems of holomorphic almost periodic equations. (Russian) Uspekhi Mat. Nauk 39 (1984), no. 1(235), 123-124. MR733961 (86a:32007)

[5] A. Khovanskii, Newton polyhedra, a new formula for mixed volume, product of roots of a system of equations, Fields Inst. Comm., Vol. 24 (1999), 325-364. MR1733583 (2001d:52013)

[6] A. Khovanskii, Fewnomials, A.M.S., 1991 MR1108621 (92h:14039)

[7] A. Khovanskii, S. Yakovenko, Generalized Rolle Theorem in $\mathbb{R}^{n}$ and $\mathbb{C}$, J. Dynam. Control Systems 2 (1996), no. 1, 103-123. MR1377431 (97f:26016)

[8] J. Ritt, On the zeros of exponential polynomials, Transactions of The American Mathematical Society, Vol. 31, Issue 4 (1929), 680-686. MR1501506

[9] E. Soprunova, Zeros of systems of exponential sums and trigonometric polynomials, Moscow Mathematical Journal, Vol. 6 (2006), no. 1. MR2265953

Department of Mathematical Sciences, Kent State University, Kent, Ohio 44242

E-mail address: soprunova@math.kent.edu 